\title{
A Multiphysics Simulation of a Healthy and a Diseased Abdominal Aorta
}

\author{
Robert H.P. McGregor ${ }^{1}$, Dominik Szczerba ${ }^{1}$, and Gábor Székely ${ }^{1}$ \\ Computer Vision Laboratory, Sternwartstr. 7, 8092 Zürich, Switzerland \\ http://www.vision.ee.ethz.ch
}

\begin{abstract}
Abdominal Aortic Aneurysm is a potentially life-threatening disease if not treated adequately. Its pathogenesis is complex and multifactorial and is still not fully understood. Many biochemical and biomechanical mechanisms have been identified as playing a role in the formation of aneurysms but it is as yet unclear what triggers the process. We investigated the role of the relevant biomechanical factors, in particular the wall shear stress and the intramural wall stress by simulating fluid structure interaction between the blood flow and the deforming arterial wall in a healthy abdominal aortic bifurcation, the preferred location of the disease. We then extended this study by introducing a hypothetical weakening of the aortic wall. Intramural wall stress was considerably higher and wall shear stress considerably lower in this configuration, supporting the hypothesis that biomechanical aneurysmal growth factors are self-sustaining.
\end{abstract}

\section{Introduction}

\subsection{Background}

Abdominal aortic aneurysm (AAA) is recognized as a major cause of mortality in developed countries. Fifteen thousand people die every year from AAA rupture in the United States alone, making it the 13th leading cause of death in this country [1. It is characterized by a permanent and irreversible widening of the infrarenal abdominal aorta, which, if left untreated, can dilate further and eventually rupture, leading to death in most cases. Although this disease is increasingly common due to ageing population, its precise causes are still not exactly understood. It is generally believed that there is no single cause for its occurrence, but AAA results from a complex interaction of many biochemical and biomechanical processes in which genetic predispositions also play a part. The observation that it often occurs simultaneously with atherosclerosis led to the assumption that it is linked to this disease, although this theory has been challenged recently 2. Nevertheless, from a biomechanical point of view, the study of both these pathologies requires precise knowledge of the local hemodynamic factors acting on the arterial wall as well as the stresses within it. Until recently such a holistic approach has been difficult to implement, due to limitations of imaging equipment and in particular of computing power. Thanks to

N. Ayache, S. Ourselin, A. Maeder (Eds.): MICCAI 2007, Part II, LNCS 4792, pp. $227-2342007$.

(C) Springer-Verlag Berlin Heidelberg 2007 
recent technological advances we are now able to perform full fluid-solid interaction (FSI) simulations of the living artery with commercially available hardware, thus gaining valuable insight into these processes.

\subsection{Related Work}

The pathogenesis of AAA is subject of ongoing research. A recent recapitulative study in this area is presented by Ailawadi et al. [3], who point out the complexity of the disease and identify the main mechanisms participating in AAA formation. They agree with Grange et al. 2] to say that the wall structure and elasticity is changed in a diseased artery as compared to a healthy one mostly due to degradation of elastin and collagen, the two principal load-bearing fibers of the extracellular matrix, by biochemical processes.

There is a large number of publications pertaining to the elasticity and deformation model of the arterial wall. Zhao et al. 4] present a good overview of this topic and show the large diversity of approaches. Every author seems to develop a new mathematical model of his own, making it very difficult to choose a particular one from the literature. Of special interest to us is the contribution by Raghavan et al. 5] who compare the elastic properties of an aneurysmal arterial wall with those of a healthy one and link the elastin and collagen contributions to an overall constitutive equation.

Raghavan expands and uses this model in subsequent work [6] for simulating the intramural wall stress (IWS) in AAAs, using patient specific geometries and static pressure-loading. Much research is taking place in this direction, the main idea being to develop a useful clinical tool to assess an AAA's risk of rupture as the current indicators (aneurysmal diameter or, more recently, volume) are considered inexact, see [7] for an overview. Leung et al. 8] went further and performed a fully coupled FSI simulation to evaluate the rupture risk, but concluded that considering the amount of computational power and time involved, and the small difference in results, static models were already sufficient for this kind of analysis.

AAA is known to preferentially occur distal to the renal arteries and proximal to the arterial bifurcation. This has led to the hypothesis that the complex flow patterns occurring here are a possible cause, in particular that low timeaveraged wall shear stress (WSS), flow recirculation as well as high temporal gradients of wall shear stress are important factors in atheroscopic plaque formation and aneurysmal genesis. Several researchers have simulated blood flow at the abdominal bifurcation so as to quantify these effects, e.g. 9], but they do not account for the biomechanical effects inside the wall.

In fact, there is little to be found in the literature on IWS in a healthy aorta. Zhao et al. 4] have reviewed the available tools for FSI simulations and presented their own method applied to model a carotid bifurcation.

FSI simulations are now becoming more accessible due to the technological progress of computer hardware and in particular the availability of 64 bit architectures, offering increased memory. We propose to use the opportunities which these improved conditions open to closer investigate and analyze the 
biomechanical processes inherent to aneurysmal growth, using a holistic approach. The novelty of our study lies in the investigation a healthy patientspecific artery using FSI and to model its long-term weakening based on biomechanical factors.

\section{Methods}

Workflow pipeline. We start the procedure by acquiring the MRI data from which the arterial lumen is segmented. This geometry is then filled with a tetrahedral mesh and prisms are extruded to create the wall mesh. A FSI simulation is then performed to model the behavior of a healthy artery throughout a heart cycle. Finally a wall weakening is introduced to study the long-term behavior of a diseased aorta.

The MRI data were acquired using a Philips Achieva 1.5T, using a $3-\mathrm{D}$ phase contrast (PC) pulse sequence, which provided a time-resolved velocity field as well as bright blood anatomy images of the lower abdomen of a healthy 35 year old male volunteer. The slices are taken perpendicularly to the infrarenal abdominal aorta. The images are $224 \times 207$ pixels in size for 20 slices, with a slice thickness of $5 \mathrm{~mm}$ and an in-plane pixel spacing of $1.29 \mathrm{~mm}$, over 24 phases of the cardiac cycle. These data were recorded in 18 min (pure measurement time) and the volunteer had to perform breath holds during the acquisition cycles. The velocity data were used as a reference for validation of the simulated blood flow.

The arterial lumen was segmented from the anatomy images and smoothed, so as to produce an initial surface mesh. This was then used as input to a novel meshing algorithm [10] resulting in a high quality tetrahedral mesh with refinement close to the walls. Three layers of $0.5 \mathrm{~mm}$ thick prisms were then added to the surface, to model the aortic wall as this is typically $\sim 1.5 \mathrm{~mm}$ thick 8]. These prisms were then subdivided into tetrahedra. Figure 1(a) shows a cutaway of the final mesh. The refinement of the lumen mesh is necessary for two reasons: firstly it speeds up computations enormously and secondly it ensures a sufficiently high resolution at the interface. This is desirable as the wall is thin, but still needs to have at least three layers of cells for reliable predictions. In order to have wall elements which are not excessively thin radially, they need to have short edges in the circumferential direction. The final lumen mesh consists of 10'043 tetrahedra with an average quality of 0.74 (defined as normalized radius ratio of circumscribed to inscribed sphere), while the wall mesh contains 25'686 tetrahedra with an average quality 0.41 .

The simulations were performed by a finite element model (FEM) code, solving the incompressible Navier-Stokes equations coupled with the structural mechanics stress equations and using an Arbitrary Lagragian Eulerian (ALE) model for mesh displacement. All the mesh elements were chosen to be Lagrangian quadratic. The coupling is passive in the sense that we do not address momentum transfer from constricting walls. The geometry was fixed at both inlet and outlets and a sinusoidal pressure wave, mimicking the systolic and diastolic pressure distributions was prescribed as boundary conditions for the flow equations. 
The flow was assumed to be laminar, incompressible and Newtonian (an assumption which has shown to be valid for large arteries [1]), with a density of 1020 $\mathrm{kg} / \mathrm{m}^{3}$ and a dynamic viscosity of $0.003 \mathrm{~Pa} / \mathrm{s}$. The arterial wall was considered to be linear elastic as this has shown to be a valid approximation for pressure loading within normal systolic and diastolic ranges [12, with an elasticity modulus of $2.710^{6} \mathrm{~Pa}$, a density of $2000 \mathrm{~kg} / \mathrm{m}^{3}$ and a poisson ratio of 0.49 , so almost incompressible.

In aneurysmal tissue the extra-cellular matrix has been observed to be degraded. One hypothesis for this degradation which has been proposed by Vorp et al. 7] is that this is due to large stress over a period of time. From a biochemical point of view this corresponds to saying that the elastin and collagen, the main components of the extra-cellular matrix, are degraded when they are under load, leading to long term plastic behavior of the aorta. We simulated this by locally decreasing the Young's modulus in the infrarenal abdominal aorta above the bifurcation, corresponding to the observed preferential localization of the disease. We incrementally weakened the arterial wall so as to observe the effect this would have on WSS and IWS. The localization was governed by an upside-down Gaussian bell, which was multiplied with the weakening factor:

$$
E=E_{0}(1-w) e^{\frac{-\left(z-z_{0}\right)^{2}}{2 \sigma_{z}^{2}}},
$$

with $E_{0}$ being the original Young's modulus, $w$ the weakening ( $w=0.1$ would mean the minimal $E$ would be $10 \%$ smaller than $E_{0}$ ), $z_{0}$ was set as the midplane between the renal arteries and the iliac bifurcation and $\sigma_{z}$ was chosen to be $30 \%$ of the geometry's full length.

The use of a Gaussian bell can be justified by the propagative nature of the weakening process, which is internally driven by failure of the strengthening fibers in the extra-cellular matrix. Fibers adjacent to a broken one will suffer from increased strain and thus have a high likelihood of failing as well. In a first order approximation we model this propagation as diffusion, but a more sophisticated model could be easily integrated.

\section{$3 \quad$ Results}

Simulation of a Healthy Aortic Bifurcation. The simulation of a healthy aortic bifurcation revealed that time averaged WSS was low on the outer wall of the left branch at the bifurcation (see figure 3(c), , which is the one with the larger takeoff angle. This correlates well with the typical location of atherosclerotic plaques, but it is also interesting to observe that the upper portion of the geometry (proximal to the bifurcation), especially on the anterior side, also suffers from low time averaged WSS (see figure $3(\mathrm{~b})$. It may also be observed that the aorta proximal to the bifurcation has a higher IWS than in the iliac arteries (see figures $3(\mathrm{e})$ and 3(f) and this holds throughout the cardiac cycle (see figure1(b)). This region is typically prone to AAA and the causes may lie in a combination of low time averaged WSS, causing atherosclerotic plaques which locally modify 


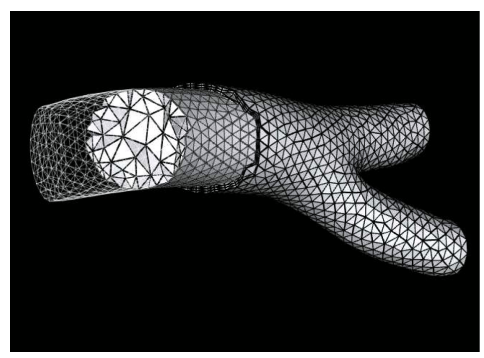

(a)

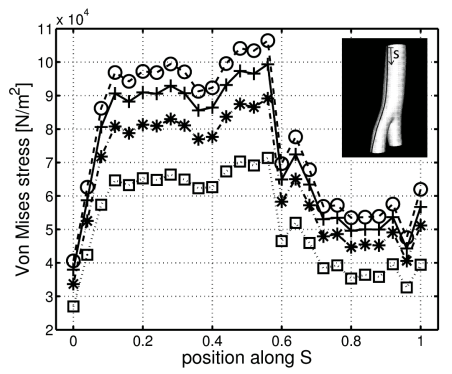

Fig. 1. (a) Cutaway of the mesh used for simulation. (b) IWS stress along line $s$ at different phases of the cardiac cycle: + : during systolic acceleration, $\circ$ : at peak systole *: during diastolic deceleration and $\square$ : at peak flow reversal.

the wall's mechanical properties and high time averaged IWS, causing fatigue in the elastin and collagen fibres which may eventually become degraded. The use of a coupled model also allows for the computation of displacements of the aortic walls during the heart cycle. The maximal displacements were observed at the bifurcation (see figure $3(\mathrm{a})$, in the locations where the IWS is largest. The computed displacements are 4.3 times greater at these locations than the average wall displacement, being up to $1.02 \mathrm{~mm}$ at peak systole. This localized concentration of high IWS is very similar to what Thubrikar et al. 13] found at arterial branchings.

Simulating Aneurysmal Growth. The weakening $(w)$ was incrementally increased until reaching a maximum of $96 \%$. The results are shown in figure 4 . Figure 4(b) shows the IWS in the weakened aorta (with maximal weakening) at peak systole, this is on average 1.54 times larger than in the healthy aorta, the most noticeable differences being on the posterior wall. Despite the smaller Young's modulus, the stresses are clearly higher in a weakened artery, this is in agreement with Leung [8] and Fillinger et al. 14] who also find higher average stress in the aneurysmal area than in the healthy one. The WSS (see figure $4(\mathrm{~b})$ is on average 0.74
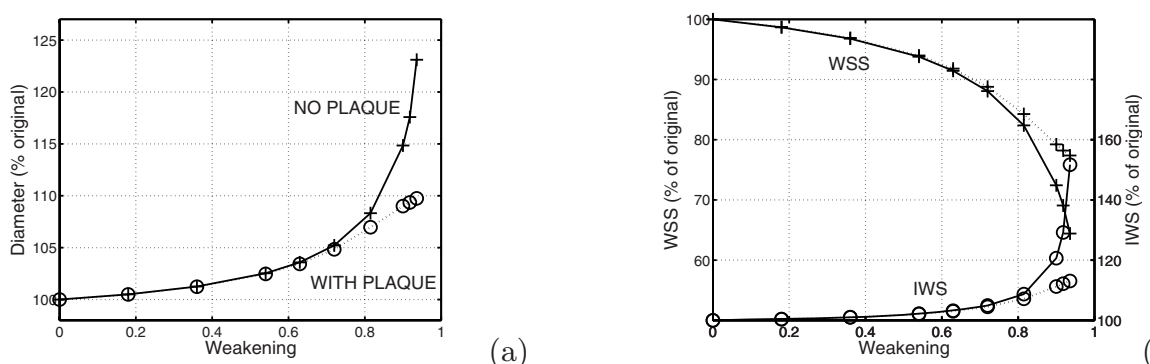

Fig. 2. The effect of weakening on the arterial wall: (a) increase in artery diameter, (b) average IWS and average WSS. In both cases the dotted line shows the effect of plaque formation and the solid one ignores it. 


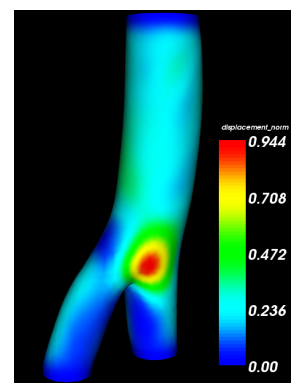

(a) Absolute displacement of wall at peak systole (in $\mathrm{mm}$ ).

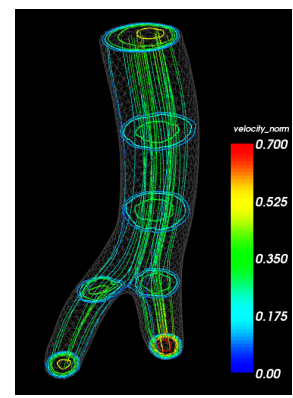

(d) Velocity profile at systolic peak (in $\mathrm{m} / \mathrm{s})$.

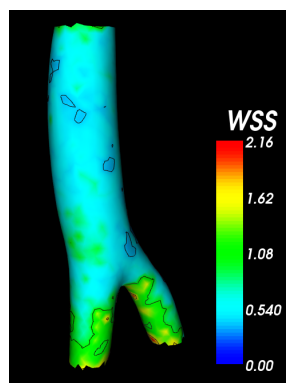

(b) Time averaged WSS, anteriorposterior view (in $\left.N / m^{2}\right)$.

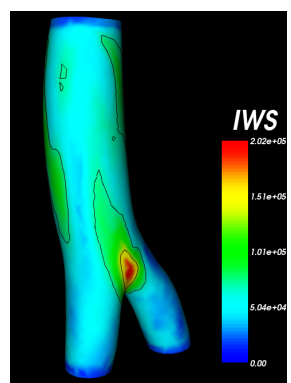

(e) Time averaged IWS (in $N / m^{2}$ ), anterior-posterior view.

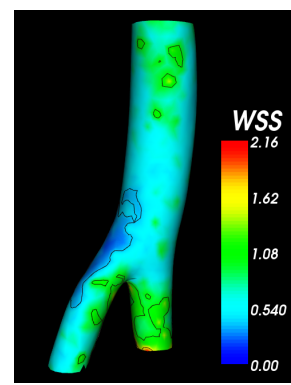

(c) Time averaged WSS, posterioranterior view (in $\left.N / m^{2}\right)$.

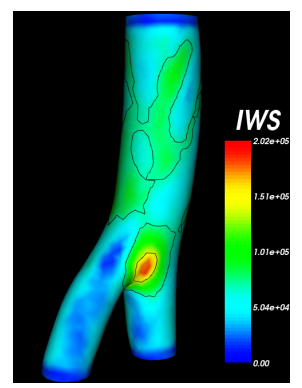

(f) Time averaged IWS (in $N / m^{2}$ ), posterior-anterior view.

Fig. 3. Results of simulation

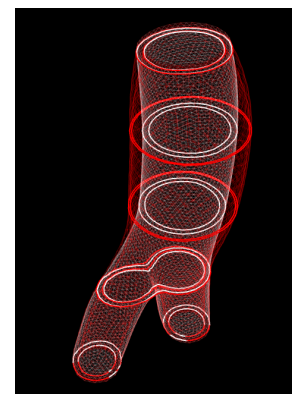

(a) Weakened artery deformation as compared to healthy one.

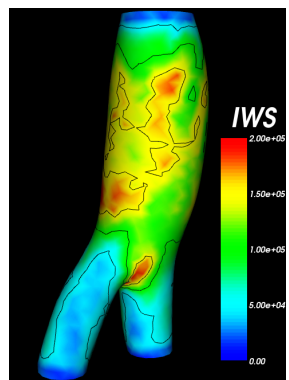

(b) IWS at peak systole, posterioranterior view.

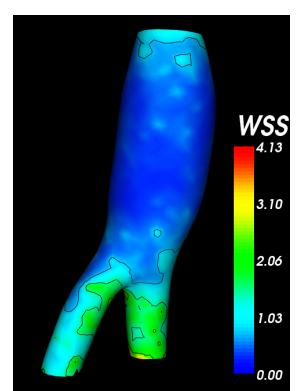

(c) WSS at peak systole, posterioranterior view.

Fig. 4. Results of simulation using a weakened artery 
times that in a healthy aorta. Figure 2(b) shows the evolution of these factors as a function of weakening of the aorta. Assuming WSS and IWS are factors which degrade the wall, they are also factors which worsen with wall decay, suggesting that the aortic wall weakening will lead to a snowball effect. When considering the results shown on figure 2 (solid lines), one would expect that an aneurysm is doomed to rupture within a short delay, due to the exponential nature of the process. However, this does not take into account the formation of atherosclerotic plaques in the intima, which is caused by low time averaged WSS and contributes to stiffening the wall. We performed a study of the effect this would have by adding a term to the Young's modulus which increased in areas of low WSS. As figures 2 (dotted lines) show, this indeed stabilizes the weakening process to some extent.

\section{Validation}

Validation of such a simulation is always difficult, as there is no in vivo gold standard. We are, however, able to compare the simulated flow velocity to that measured by MRI, even though this also has an inherent error. We found that our computed velocity profile (see figure $3(\mathrm{~d})$ is qualitatively similar to the MRI profile, even though the latter is rather noisy especially at low velocities (flow reversal). Quantitatively it is hard to compare the two as we rely on a hypothetical pressure profile, which corresponds to average physiological values, but does not necessarily match that of the actual volunteer. We therefore prefer to compare our findings with generic results of other researchers active in this field. We find similar flow profiles as Long et al. 9] and Taylor et al. 15] and we are also in quantitative and qualitative agreement regarding the time averaged WSS distributions. Zhao et al. 4 present the stress distribution in a healthy abdominal aortic bifurcation and, although they use a static model and lower pressure load, the spatial variation they find is very similar to ours.

\section{Conclusions}

We have performed a full FSI simulation of a healthy aortic bifurcation with physiological loading. We have shown that both low WSS and high IWS are to be found preferentially in the aorta proximal to the bifurcation and distal to the renal arteries, which correlates well with the typical positioning of AAAs. We have also shown that using the same model, but with a locally weakened wall, the biomechanical factors contributing to aneurysm growth are worsened, thus causing a snowball effect which to continual growth of the aneurysm and ultimately to rupture. However, we were able to demonstrate that atherosclerotic plaque formation counteracts this effect and may help to reach an equilibrium in the process. In the future we hope that such a tool could be used to identify risk factors and eventually to predict the onset and the evolution of the disease. 
Acknowledgments. This work was supported by the Indo-Swiss Joint Research Programme.

\section{References}

1. Gillum, R.F.: Epidemiology of aortic aneurysm in the united states. Journal of Clinical Epidemiology 48(11), 1289-1298 (1995)

2. Grange, J.J., Davis, V., Baxter, B.T.: Pathogenesis of abdominal aortic aneurysm: an update and look toward the future. Cardiovascular Surgery 5(3), 256-265 (1997)

3. Ailawadi, G., Eliason, J.L., Upchurch, G.R.: Current concepts in the pathogenesis of abdominal aortic aneurysm. Journal of Vascular Surgery 38(3), 584-588 (2003)

4. Zhao, S.Z., Xu, X.Y., Collins, M.W.: The numerical analysis of fluid-solid interactions for blood flow in arterial structures part 1: a review of models for arterial wall behaviour. Proceedings of the Institution of Mechanical Engineers, Part H: Journal of Engineering in Medicine V212(4), 229-240 (1998)

5. Raghavan, M.L., Webster, M.W., Vorp, D.A.: Ex vivo biomechanical behavior of abdominal aortic aneurysm: Assessment using a new mathematical model. Ann. Biomed. Eng. 24(5), 573-582 (1996)

6. Raghavan, M.L., Vorp, D.A., Federle, M.P., Makaroun, M.S., Webster, M.W.: Wall stress distribution on three-dimensionally reconstructed models of human abdominal aortic aneurysm. Journal of Vascular Surgery 31(4), 760-769 (2000)

7. Vorp, D.A., Geest, J.P.V.: Biomechanical determinants of abdominal aortic aneurysm rupture. Arterioscler. Thromb. Vasc. Biol. 25(8), 1558-1566 (2005)

8. Leung, J.H., Wright, A.R., Cheshire, N., Crane, J., Thom, S.A., Hughes, A.D., Xu, Y.: Fluid structure interaction of patient specific abdominal aortic aneurysms: a comparison with solid stress models. BioMedical Engineering OnLine 5(33) (2006)

9. Long, Q., Xu, X.Y., Bourne, M., Griffith, T.M.: Numerical study of blood flow in an anatomically realistic aorto-iliac bifurcation generated from MRI data. Magnetic Resonance in Medicine 43(4), 565-576 (2000)

10. Szczerba, D., McGregor, R., Szekely, G.: High quality surface mesh generation for multi-physics bio-medical simulations. In: Simulation Of Multiphysics Multiscale Systems, 4th International Workshop (2007)

11. Berger, S.A., Jou, L.D.: Flows in stenotic vessels. Annual Review of Fluid Mechanics $32(1), 347-382$ (2000)

12. Leuprecht, A., Perktold, K., Prosi, M., Berk, T., Trubel, W., Schima, H.: Numerical study of hemodynamics and wall mechanics in distal end-to-side anastomoses of bypass grafts. Journal of Biomechanics 35(2), 225-236 (2002)

13. Thubrikar, M.J., Roskelley, S.K., Eppink, R.T.: Study of stress concentration in the walls of the bovine coronary arterial branch. Journal of Biomechanics 23(1), 15-17 (1990)

14. Fillinger, M.F., Marra, S.P., Raghavan, M.L., Kennedy, F.E.: Prediction of rupture risk in abdominal aortic aneurysm during observation: Wall stress versus diameter. Journal of Vascular Surgery 37(4), 724-732 (2003)

15. Taylor, C.A., Hughes, T.J.R., Zarins, C.K.: Finite element modeling of threedimensional pulsatile flow in the abdominal aorta: Relevance to atherosclerosis. Annals of Biomedical Engineering V26(6), 975-987 (1998) 